\title{
Rational Design Based on Bioactive Conformation Analysis of Pyrimidinylbenzoates as Acetohydroxyacid Synthase Inhibitors by Integrating Molecular Docking, CoMFA, CoMSIA and DFT Calculations
}

\author{
Yan-Zhen He, ${ }^{1}$ Yuan-Xiang Li, ${ }^{1}$ Zhen Xi, ${ }^{2} *$ Congwei Niu, ${ }^{2}$ Jian Wan, ${ }^{1}$ Li Zhang, ${ }^{1}$ Guang-Fu Yang ${ }^{1, *}$ \\ ${ }^{1}$ Key Laboratory of Pesticide \& Chemical Biology, Ministry of Education, College of Chemistry, Central China \\ Normal University, Wuhan, Hubei 430079, P.R.China; \\ ${ }^{2}$ State Key Laboratory of Elemento-Organic Chemistry, Nankai University, Tianjin 300071, P. R. China
}

*To whom correspondence should be addressed. Fax: 86-27-67867141, E-mail: gfyang@mail.ccnu.edu.cn

(1) The constraints defined in FlexX-Pharm. The gray surfaces indicates the FlexX-Pharm surface and the interaction constraints labeled in yellow words. Three FlexX-Pharm constraints were defined as following:

A $\pi-\pi$ stacking interaction between the indolyl group of Trp586 and dimethoxypyrimidinyl was defined.

$\mathrm{NH} 1$ and $\mathrm{NH} 2$ of Arg380 were set as hydrogen bond donors.

In order to prevent the phenyl group of pyrimidinylbenzoates from stacking onto the indolyl plane of Trp586, a spatial constraint was defined delineating the essential presence of a nitrogen atom within the area of $1.5 \AA$ to the center of pyrimidinyl ring of chlorimuron ethyl.

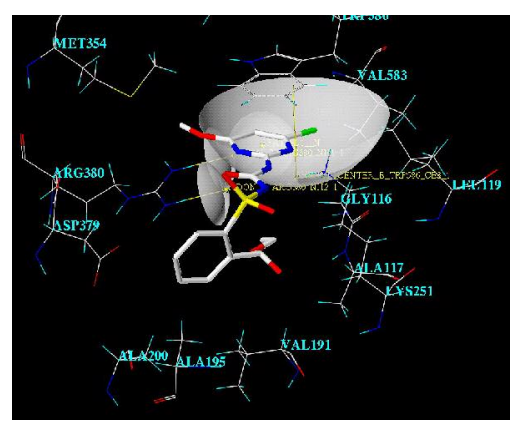


(2) The results of FlexX-Pharm docking of all successful docking molecules in the binding site (A).

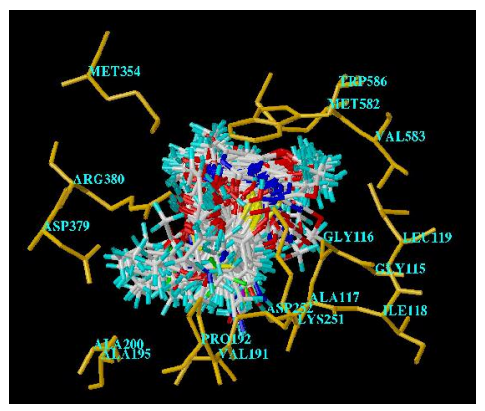

(3) The conformations of Compound 12(B, left-extending) and Compound 31(C, right-extending) with the highest Total Score.

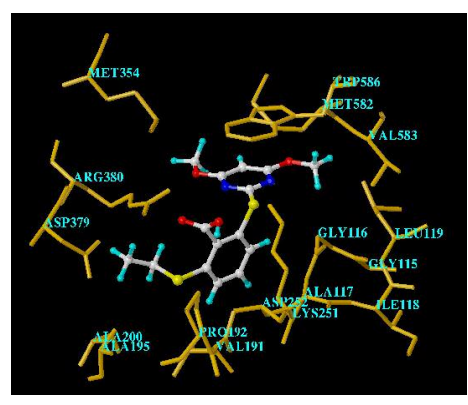

A

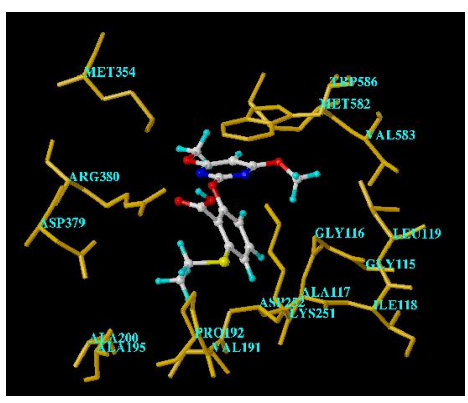

B

(4) The alignment results based on docking conformation (A), DFT-optimized conformation-L (B) and DFT-optimized conformation-R (C)

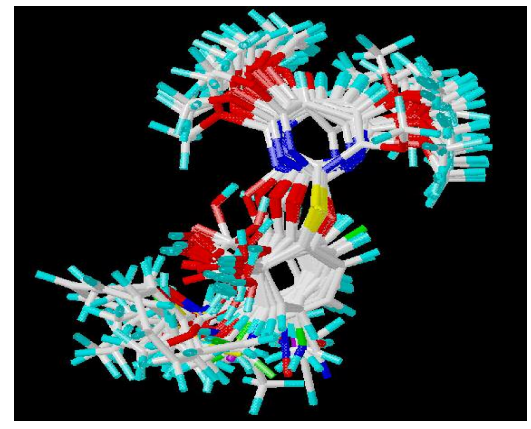

A

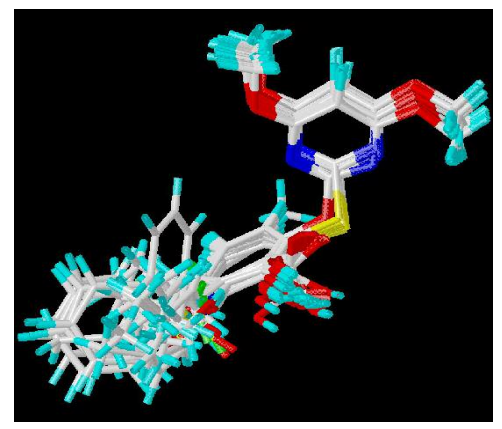

B

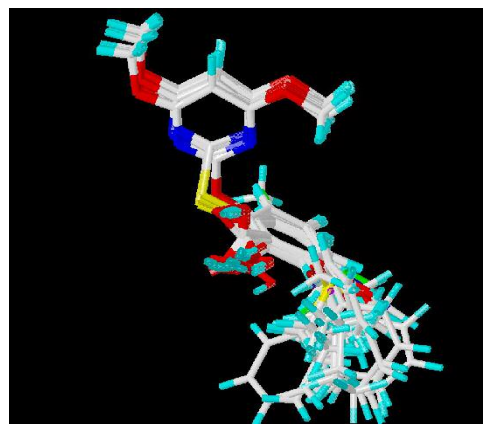

$\mathrm{C}$ 
(5)

Table 1 The effects of different types of charge on the 3D-QSAR results using the DFT-optimized conformation

\begin{tabular}{|c|c|c|c|c|c|c|c|c|}
\hline & \multicolumn{4}{|c|}{ GAST_HUCK } & \multirow{2}{*}{\multicolumn{2}{|c|}{ Model-L }} & \multirow{2}{*}{\multicolumn{2}{|c|}{ Model-R }} \\
\hline & \multicolumn{2}{|c|}{ Model-L } & \multicolumn{2}{|c|}{ Model-R } & & & & \\
\hline & CoMFA & CoMSIA & CoMFA & CoMSIA & CoMFA & CoMSIA & CoMFA & CoMSIA \\
\hline $\mathrm{q}^{2}$ & 0.547 & 0.471 & 0.668 & 0.553 & 0.589 & 0.525 & 0.742 & 0.495 \\
\hline$S_{\text {press }}$ & 0.804 & 0.860 & 0.695 & 0.790 & 0.790 & 0.824 & 0.620 & 0.849 \\
\hline$r^{2}$ & 0.748 & 0.687 & 0.883 & 0.714 & 0.955 & 0.750 & 0.936 & 0.770 \\
\hline s & 0.599 & 0.662 & 0.413 & 0.632 & 0.260 & 0.597 & 0.308 & 0.572 \\
\hline Comp. & 4 & 3 & 5 & 3 & 7 & 4 & 6 & 4 \\
\hline $\mathrm{F}$ & 36.404 & 36.595 & 72.381 & 41.694 & 140.726 & 36.835 & 115.109 & 41.090 \\
\hline \multicolumn{9}{|c|}{ Contribution } \\
\hline Ste & 0.521 & 0.148 & 0.620 & 0.210 & 0.493 & 0.207 & 0.633 & 0.228 \\
\hline Ele & 0.479 & 0.510 & 0.380 & 0.456 & 0.507 & 0.505 & 0.367 & 0.434 \\
\hline Hydro & & 0.342 & & 0.334 & & 0.289 & & 0.338 \\
\hline$r_{\text {pred }}^{2}$ & 0.8322 & & 0.8187 & 0.9086 & 0.8591 & 0.8433 & 0.798 & 0.935 \\
\hline
\end{tabular}

Table 1 Continued.

\begin{tabular}{|c|c|c|c|c|c|c|c|c|}
\hline & \multicolumn{4}{|c|}{ PM3/PM3 } & \multicolumn{4}{|c|}{ DFT/ESP } \\
\hline & \multicolumn{2}{|c|}{ Model-L } & \multicolumn{2}{|c|}{ Model-R } & \multicolumn{2}{|c|}{ Model-L } & \multicolumn{2}{|c|}{ Model-R } \\
\hline & CoMFA & CoMSIA & CoMFA & CoMSIA & CoMFA & CoMSIA & CoMFA & CoMSIA \\
\hline $\mathrm{q}^{2}$ & 0.591 & 0.410 & 0.737 & 0.415 & 0.711 & 0.555 & 0.788 & 0.720 \\
\hline$S_{\text {press }}$ & 0.772 & 0.908 & 0.625 & 0.913 & 0.656 & 0.797 & 0.562 & 0.651 \\
\hline$r^{2}$ & 0.892 & 0.680 & 0.934 & 0.760 & 0.947 & 0.810 & 0.960 & 0.921 \\
\hline $\mathrm{s}$ & 0.398 & 0.669 & 0.314 & 0.585 & 0.281 & 0.521 & 0.244 & 0.347 \\
\hline Comp. & 5 & 3 & 6 & 4 & 6 & 4 & 6 & 7 \\
\hline $\mathrm{F}$ & 78.894 & 35.455 & 110.682 & 38.809 & 140.188 & 52.269 & 188.254 & 76.218 \\
\hline \multicolumn{9}{|c|}{ Contribution } \\
\hline Ste & 0.496 & 0.209 & 0.596 & 0.241 & 0.474 & 0.203 & 0.502 & 0.244 \\
\hline Ele & 0.504 & 0.515 & 0.404 & 0.427 & 0.526 & 0.569 & 0.498 & 0.503 \\
\hline Hydro & & 0.276 & & 0.331 & & 0.228 & & 0.253 \\
\hline$r_{\text {pred }}^{2}$ & 0.7925 & 0.8192 & 0.815 & 0.947 & 0.7876 & 0.8252 & 0.8357 & 0.8794 \\
\hline
\end{tabular}


(6) The plots of the predicted $p \mathrm{IC}_{50}$ values versus the actual $p K i$ values of compounds $\mathbf{6 3} \sim \mathbf{6 8}$ (A, CoMFA model; $\mathrm{B}$, CoMSIA model)

\begin{tabular}{ccccccc}
\hline Compunds & $\mathbf{6 3}$ & $\mathbf{6 4}$ & $\mathbf{6 5}$ & $\mathbf{6 6}$ & $\mathbf{6 7}$ & $\mathbf{6 8}$ \\
\hline Tested $p$ Ki value & 3.10 & 3.44 & 3.69 & 3.68 & 3.67 & 3.36 \\
Predicted $p \mathrm{IC}_{50}$ by CoMFA model & 4.82 & 5.55 & 5.82 & 5.82 & 5.82 & 5.84 \\
Predicted $p \mathrm{IC}_{50}$ by CoMSIA model & 5.64 & 5.94 & 6.29 & 6.24 & 6.33 & 6.01 \\
\hline
\end{tabular}

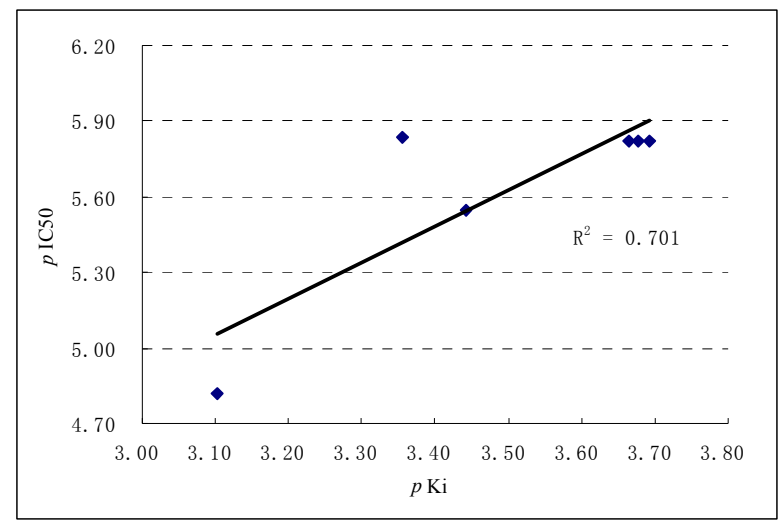

A

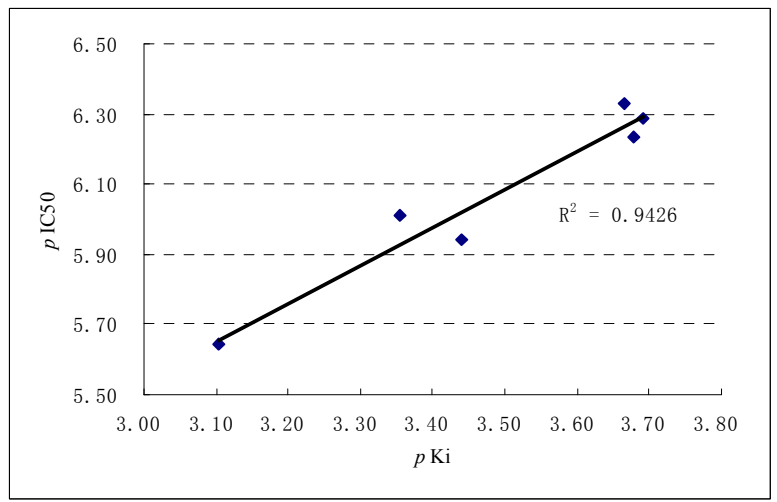

B

(7) The plots of the predicted $\mathrm{IC}_{50}$ values versus the actual $K i$ values of compounds $63 \sim 68$ (A, based on CoMFA model; B, based on CoMSIA (7) model).

\begin{tabular}{cccccc}
\hline Compunds & $\mathbf{6 3}$ & $\mathbf{6 4}$ & $\mathbf{6 5}$ & $\mathbf{6 6}$ & $\mathbf{6 7}$ \\
\hline Tested $K i$ value $\left(\times 10^{-4} \mathrm{M}\right)$ & 7.89 & 3.62 & 2.03 & 2.10 & 2.16 \\
Predicted $\mathrm{IC}_{50}$ by CoMFA model $\left(\times 10^{-6} \mathrm{M}\right)$ & 14.19 & 11.40 & 7.60 & 11.83 & 1.42 \\
Predicted $\mathrm{IC}_{50}$ by CoMSIA model $\left(\times 10^{-6} \mathrm{M}\right)$ & 16.33 & 16.56 & 16.14 & 21.23 & 1.15 \\
\hline
\end{tabular}

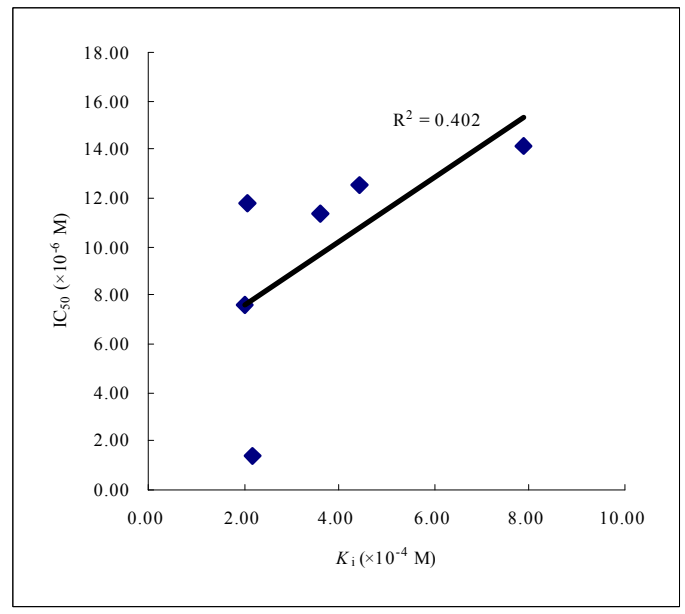

A

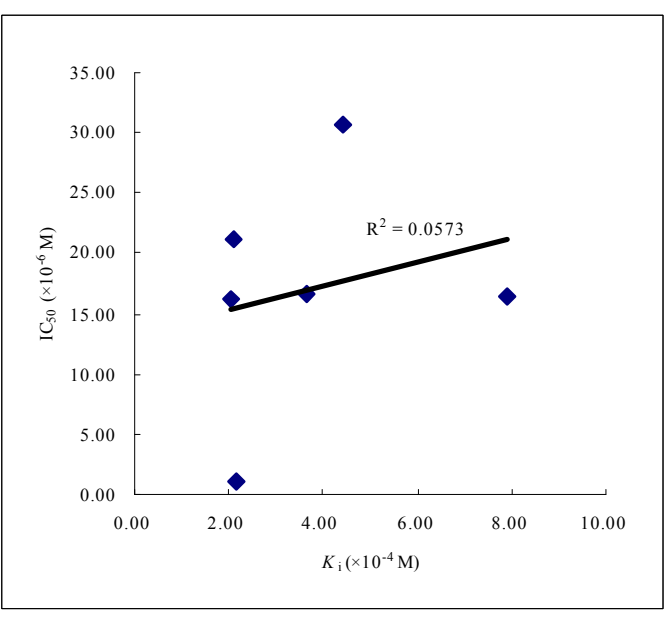

B 
(8) The plots of the predicted $\mathrm{IC}_{50}$ values versus the actual $K i$ values of compounds $\mathbf{6 3} \sim \mathbf{6 8}$ according to the 3D-QSAR models based on the alignment using docking conformations (A, CoMFA model; B, CoMSIA model).

\begin{tabular}{ccccccc}
\hline Compunds & $\mathbf{6 3}$ & $\mathbf{6 4}$ & $\mathbf{6 5}$ & $\mathbf{6 6}$ & $\mathbf{6 7}$ & $\mathbf{6 8}$ \\
\hline Tested $K i$ value $\left(\times 10^{-4} \mathrm{M}\right)$ & 7.89 & 3.62 & 2.03 & 2.10 & 2.16 & 4.41 \\
Predicted $\mathrm{IC}_{50}$ by CoMFA model $\left(\times 10^{-6} \mathrm{M}\right)$ & 0.23 & 3.30 & 3.45 & 3.25 & 3.47 & 0.18 \\
Predicted $\mathrm{IC}_{50}$ by CoMSIA model $\left(\times 10^{-8} \mathrm{M}\right)$ & 1.02 & 1.95 & 1.95 & 1.83 & 1.87 & 0.42 \\
\hline
\end{tabular}

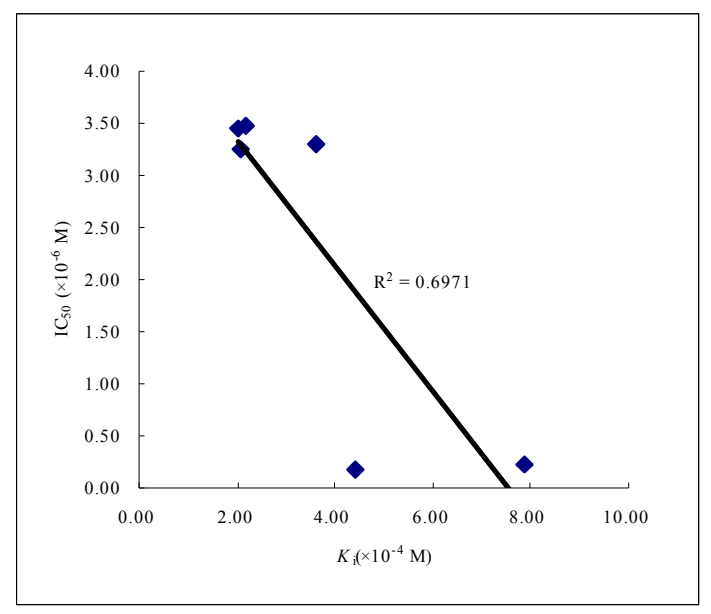

A

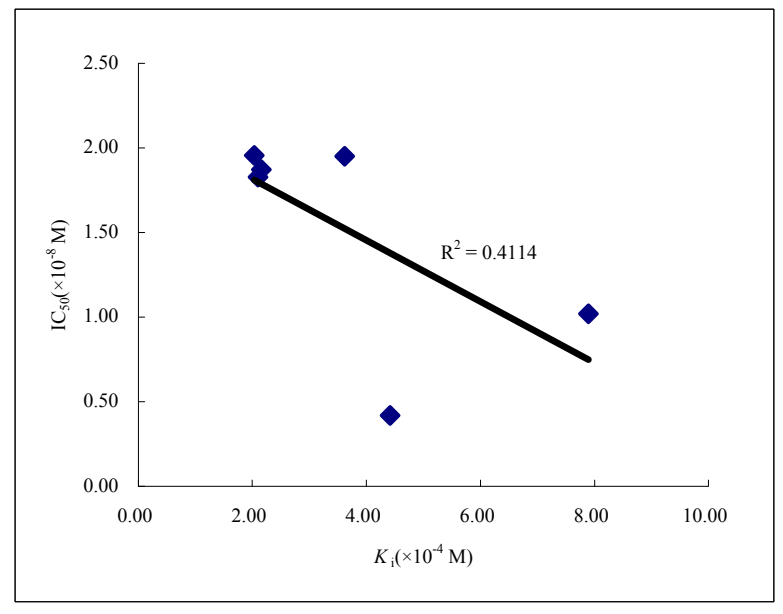

B

(9) Synthesis and characterization of compounds 63 68.<smiles></smiles>

1<smiles>COc1cc(OC)nc(S(=O)(=O)OC)n1</smiles>
acetone<smiles>COc1cccc2c1C(=O)OCC21OCCO1</smiles>

2<smiles>COc1cc(OC)nc(Oc2cccc(C(C)=N)c2C(N)=O)n1</smiles>

63

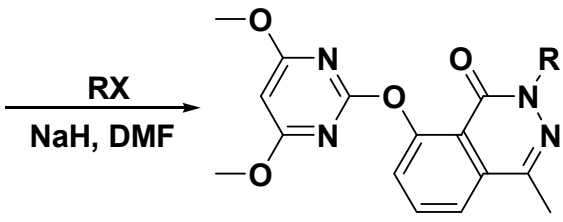

$64 \sim 68$

Scheme 1 Synthetic route of compounds $63 \sim 68$

Preparation of methyl 2-(4,6-dimethoxypyrimidin-2-yloxy)-6-(2-methyl-1,3-dioxolan-2-yl) benzoate (2). To $0.51 \mathrm{~g}$ (2.1 mmol) methyl 2-hydroxy-6-(2-methyl-1,3-dioxolan-2-yl)benzoate (1) and anhydrous $\mathrm{K}_{2} \mathrm{CO}_{3} 0.59 \mathrm{~g}(4.2 \mathrm{mmol})$ in acetone $(10 \mathrm{~mL})$ was stirred at 56 for $1 \mathrm{~h}$. 
4,6-dimethoxy-2-(methylsulfonyl)pyrimidine (DMSP) $0.49 \mathrm{~g}(2.25 \mathrm{mmol})$ was added. The reaction was continued for $10 \mathrm{~h}$. The resulting mixture was cooled to room temperature, filtered with infusorial earth and concentrated on a rotary evaporator to give crude white solid, crystalline 2 could be obtained by recrystallization from ethanol. Yield 80\%, m.p. 131-133 . ${ }^{1} \mathrm{H} \mathrm{NMR}\left(\mathrm{CDCl}_{3}\right)$ : $\delta 1.76\left(\mathrm{~s}, 3 \mathrm{H}, \mathrm{CH}_{3}\right), 3.68\left(\mathrm{~m}, 2 \mathrm{H}, \mathrm{CH}_{2}\right), 3.78\left(\mathrm{~s}, 3 \mathrm{H}, \mathrm{COOCH}_{3}\right), 3.90\left(\mathrm{~s}, 6 \mathrm{H}, \mathrm{OCH}_{3} \times 2\right), 3.97(\mathrm{~m}$, $\left.2 \mathrm{H}, \mathrm{CH}_{2}\right), 5.75$ (s, 1H, PyHet-H), 7.16-7.40 (m, 3H, Ar-H).

Preparation of 8-(4,6-dimethoxypyrimidin-2-yloxy)-4-methylphthalazin-1(2H)-one (63). To a $250 \mathrm{~mL}$ round-bottom flask, $3.0 \mathrm{~g}$ of $(7.9 \mathrm{mmol})$ methyl 2-(4,6-dimethoxypyrimidin-2-yloxy)-6(2-methyl-1,3-dioxolan-2-yl)benzoate (2), $1.26 \mathrm{~g}$ (11.9 mmol) of hydrazine hydrochloride and 200 $\mathrm{mL}$ of methanol were added gradually. The resulting mixture was stirred at room temperature for 24 $\mathrm{h}$ and then concentrated on a rotary evaporator; the crude product obtained was poured into $300 \mathrm{~mL}$ of water and extracted with chloroform $(100 \mathrm{~mL} \times 3)$. The combined chloroform extracts were washed with brine $(100 \mathrm{~mL} \times 2)$, dried with anhydrous magnesium sulfate, filtered off by suction and the solvent was evaporated to give crude, white crystals 63 which could be purified by recrystallization from toluene. Yield 88\%. m.p. 225-227 . ${ }^{1} \mathrm{H} \mathrm{NMR}\left(\mathrm{CDCl}_{3}\right): \delta 2.58\left(\mathrm{~s}, 3 \mathrm{H}, \mathrm{CH}_{3}\right)$, $3.77\left(\mathrm{~s}, 6 \mathrm{H}, 2 \times \mathrm{OCH}_{3}\right), 5.75$ (s, 1H, PyHet-H), 7.53 (d, $\left.J=8.0 \mathrm{~Hz}, 1 \mathrm{H}, 7^{`}-\mathrm{ArH}\right), 7.67$ (d, J=8.0 Hz, 1H, $\left.5^{\prime}-\mathrm{ArH}\right), 7.86$ (t, $\left.J=8.0 \mathrm{~Hz}, 1 \mathrm{H}, 6{ }^{`}-\mathrm{ArH}\right), 9.56$ (s, 1H, N-H). EI MS: $m / z(\%) 314\left(\mathrm{M}^{+}, 100\right)$, 299 (40), 283 (75), 256 (16), 226 (45), 173 (21), 144 (12), 101 (13). Anal. Calcd for $\mathrm{C}_{15} \mathrm{H}_{14} \mathrm{~N}_{4} \mathrm{O}_{4}$ : C, 57.32; H, 4.49; N, 17.83. Found: C, 57.06; H, 4.32; N, 17.62.

General Procedure for the Synthesis of Target Compounds 64 68. A solution of 63 (1 mmol), $\mathrm{NaH}(60 \%$, dispersion in mineral oil) $(1.2 \mathrm{mmol})$ in $\mathrm{N}, \mathrm{N}$-dimethylformamide (DMF) $(10 \mathrm{~mL})$ was stirred at r.t. for $0.5 \mathrm{~h}$, followed by the addition of halogenated compounds $(1.1 \mathrm{mmol})$. The reaction was continued for $9-24 \mathrm{~h}$ at room temperature. The mixture was poured into $200 \mathrm{~mL}$ of water, extracted with ethyl acetate $(50 \mathrm{~mL} \times 3)$, dried with anhydrous magnesium sulfate, filtered off by suction and the solvent was evaporated to give the crude product followed by purification by chromatography on silica using petroleum ether / ethyl acetate as eluent to give the target compounds.

Data for 64. Yield, 59\%; mp 163-164 . ${ }^{1} \mathrm{H}$ NMR (400 MHz, $\left.\mathrm{CDCl}_{3}\right): \delta 2.58\left(\mathrm{~s}, 3 \mathrm{H}, \mathrm{CH}_{3}\right), 3.76(\mathrm{~s}$, $\left.6 \mathrm{H}, 2 \times \mathrm{OCH}_{3}\right), 5.36\left(\mathrm{~s}, 2 \mathrm{H}, \mathrm{CH}_{2}\right), 5.73$ (s, 1H, PyHet-H), 6.83-7.86 (m, 7H, 7ph-H). EI MS: m/z (\%) 485([M+2] $\left.]^{+}, 2\right), 483\left(\mathrm{M}^{+}, 2\right), 403$ (76), 297 (9), 249 (24), 171 (96), 169 (100), 139 (37), 89 (66). Anal. Calcd for $\mathrm{C}_{22} \mathrm{H}_{19} \mathrm{BrN}_{4} \mathrm{O}_{4}$ : C, 54.67; H, 3.96; N, 11.59. Found: C, 54.58; H, 4.21; N, 11.31 .

Data for 65. Yield, 61\%; mp 186-188 . ${ }^{1} \mathrm{H}$ NMR (400 MHz, $\left.\mathrm{CDCl}_{3}\right): \delta 2.29$ (s, 3H,), 2.57 (s, 3H, $\left.\mathrm{CH}_{3}\right), 3.71\left(\mathrm{~s}, 6 \mathrm{H}, 2 \times \mathrm{OCH}_{3}\right), 5.21\left(\mathrm{~s}, 2 \mathrm{H}, \mathrm{CH}_{2}\right), 5.76$ (s, 1H, PyHet-H), 7.21-7.83 (m, 7H, 7ph-H). EI MS: $m / z(\%) 440\left([\mathrm{M}+1]^{+}, 5\right), 439\left(\mathrm{M}^{+}, 7\right), 438\left([\mathrm{M}-1]^{+}, 18\right), 300(82), 284(85), 258(56), 256$ 
(100), 230 (53), 125 (46). Anal. Calcd for $\mathrm{C}_{22} \mathrm{H}_{19} \mathrm{ClN}_{4} \mathrm{O}_{4}$ : C, 60.21; H, 4.36; Cl, 8.08; N, 12.77. Found: C, 60.09; H, 4.64; N, 12.53 .

Data for 66. Yield, 93\%; mp 186-188 . ${ }^{1} \mathrm{H}$ NMR (400 MHz, $\left.\mathrm{CDCl}_{3}\right): \delta=2.58\left(\mathrm{~s}, 3 \mathrm{H}, \mathrm{CH}_{3}\right), 3.76$ $\left(\mathrm{s}, 6 \mathrm{H}, 2 \times \mathrm{OCH}_{3}\right), 5.39\left(\mathrm{~s}, 2 \mathrm{H}, \mathrm{CH}_{2}\right), 5.73(\mathrm{~s}, 1 \mathrm{H}$, PyHet-H), 6.89-7.87 (m, 7H, 7ph-H). EI MS: m/z (\%) 439( $\left.\mathrm{M}^{+}, 2\right), 438$ ([M-1] $\left.]^{+}, 1\right), 404$ (17), 403 (98), 300 (28), 284 (49), 258 (30), 256 (50), 230 (24), 139 (37), 125 (100), 89 (28). Anal. Calcd for $\mathrm{C}_{22} \mathrm{H}_{19} \mathrm{ClN}_{4} \mathrm{O}_{4}$ : C, 60.21; H, 4.36; N, 12.77. Found: C, 60.51; H, 4.63; N, 12.49 .

Data for 67. Yield, 89\%; mp $178 \sim 180 \quad .{ }^{1} \mathrm{H}$ NMR (400 MHz, $\left.\mathrm{CDCl}_{3}\right): \delta 2.59\left(\mathrm{~s}, 3 \mathrm{H}, \mathrm{CH}_{3}\right), 3.71$ $\left(\mathrm{s}, 6 \mathrm{H}, 2 \times \mathrm{OCH}_{3}\right), 5.22\left(\mathrm{~s}, 2 \mathrm{H}, \mathrm{CH}_{2}\right), 5.76(\mathrm{~s}, 1 \mathrm{H}$, PyHet-H), 7.20-7.84 (m, 7H, 7ph-H). EI MS: $m / z$ (\%) $\left.440\left([\mathrm{M}+1]^{+}, 6\right), 439\left(\mathrm{M}^{+}, 6\right), 438(\mathrm{M} \mathrm{-1})^{+}, 19\right), 300$ (57), 284 (57), 258 (41), 256 (90), 230 (45), 139 (47), 125 (100), 89 (29). Anal. Calcd for $\mathrm{C}_{22} \mathrm{H}_{19} \mathrm{ClN}_{4} \mathrm{O}_{4}$ : C, 60.21; H, 4.36; N, 12.77. Found: C, $60.02 ; \mathrm{H}, 4.61 ; \mathrm{N}, 12.51$.

Data for 68. Yield, 93\%; mp 156-158 . ${ }^{1} \mathrm{H}$ NMR (400 MHz, $\mathrm{CDCl}_{3}$ ): $\delta 0.90$ (t, $J=7.2 \mathrm{~Hz}, 3 \mathrm{H}$, $\left.\left(\mathrm{CH}_{2}\right)_{3} \mathrm{CH}_{3}\right), 1.31\left(\mathrm{~m}, 2 \mathrm{H},\left(\mathrm{CH}_{2}\right)_{3} \mathrm{CH}_{3}\right), 1.71\left(\mathrm{~m}, 2 \mathrm{H},\left(\mathrm{CH}_{2}\right)_{3} \mathrm{CH}_{3}\right), 2.60\left(\mathrm{~s}, 3 \mathrm{H}, \mathrm{CH}_{3}\right), 3.77(\mathrm{~s}, 6 \mathrm{H}, 2$ $\left.\times \mathrm{OCH}_{3}\right), 4.07\left(\mathrm{t}, J=7.6 \mathrm{~Hz}, 2 \mathrm{H},\left(\mathrm{CH}_{2}\right)_{3} \mathrm{CH}_{3}\right), 5.75$ (s, $1 \mathrm{H}$, PyHet-H), 7.50-7.82 (m, 3H, 3ph-H). EI MS: $m / z(\%) 371\left([\mathrm{M}+1]^{+}, 6\right), 370\left(\mathrm{M}^{+}, 16\right), 355$ (91), 313 (50), 299 (41), 283 (88), 256 (100), 230 (53), 214 (37), 189 (24), 173 (70), 139 (60). Anal. Calcd for $\mathrm{C}_{19} \mathrm{H}_{22} \mathrm{~N}_{4} \mathrm{O}_{4}$ : C, 61.61; H, 5.99; N, 15.13. Found: C, 64.56; H, 6.26; N, 14.88 .

AHAS Inhibition Activity. The $K i$ values of compounds 63 68 against wild type $A$. thaliana acetohydroxyacid synthase [AHAS] ( EC 4.1.3.18) were determined according to the methods reported previously $(1,2)$, a commercial product $(\mathrm{KIH}-6127)$ was used as a control.

\section{Reference}

(1) Xi, Z.; Niu, C. W.; Li, Q. X.; Ouyang, Z.; Ban, S. R. Studies on Herbicide Design through Mutation on Herbicidal Target--Acetohydroxyacid Synthase(I). Enzyme kinetics of wild type and mutants of E. coli AHAS II. Chinese J. Pestic. Sci. 2005, 7(3), 215-220.

(2) Chang A.K, and Duggleby R.G, Expression, purification and characterization of Arabidopsis thaliana acetohydroxyacid synthase. Biochem. J. 1997, 327, 161-169. 